\title{
Evidence and control of bifurcations in a respiratory system
}

\author{
Matías A. Goldin ${ }^{\text {a) }}$ and Gabriel B. Mindlin \\ Laboratorio de Sistemas Dinámicos, IFIBA y Departamento de Física, Facultad de Ciencias Exactas y \\ Naturales, Universidad de Buenos Aires, Pabellón 1, Ciudad Universitaria, Buenos Aires, Argentina
}

(Received 13 September 2013; accepted 10 December 2013; published online 26 December 2013)

\begin{abstract}
We studied the pressure patterns used by domestic canaries in the production of birdsong. Acoustically different sound elements ("syllables") were generated by qualitatively different pressure gestures. We found that some ubiquitous transitions between syllables can be interpreted as bifurcations of a low dimensional dynamical system. We interpreted these results as evidence supporting a model in which different timescales interact nonlinearly. (C) 2013 AIP Publishing LLC. [http://dx.doi.org/10.1063/1.4854395]
\end{abstract}

The comprehension of motor activities that give rise to complex behavior are of great interest. A widely used animal model to study them is the one of singing birds, where respiration plays a fundamental role. Different mechanisms have been proposed to account for the dynamical origin of these motor gestures. In this work, we study the temporal signals of the pressure gestures used by domestic canaries (Serinus canaria) to perform their songs. Their analysis revealed that ubiquitous occurring transitions between syllables can be interpreted as the bifurcations of a low dimensional dynamical system. This provides evidence supporting a model where different nonlinearly interacting timescales participate.

\section{INTRODUCTION}

Singing birds are a widely used animal model to study the acquisition of complex behavior. This behavior emerges from the interaction between the output of a central nervous system and the biomechanical periphery. ${ }^{1}$ In birdsong, two motor instructions were identified as fundamental variables in models used for the generation of song. These are the intra air sac pressure and the tension in the muscles of the syrinx, the avian vocal organ. ${ }^{2}$ Different alternatives have been proposed to explain the origin of these instructions in the brain. One of them posits that the telencephalon controls every timescale in the system. ${ }^{3,4}$ In this paradigm, there is a hierarchical organization with a set of neurons acting as a central pattern generator, with subsequent parts of the pathway merely executing those commands. This is in opposition to a scheme where different timescales interact nonlinearly, which we denominate "integrative model."5-8

The "single timescale" song control model is supported from observations of sparsely coding output neurons in a telencephalic nucleus called HVC (used as a proper name), ${ }^{3,9}$ as well as experiments in which song was stretched by means of cooling this nucleus in the zebra finch (Taeniopygia guttata). ${ }^{4}$ It states that all timescales present in the song arise directly from the output signal of this nucleus.

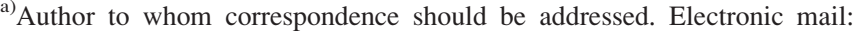
mgoldin@df.uba.ar
}

To shed light on this problem, we measured the intra air sac pressure gestures in domestic canaries (Serinus canaria), and found that some transitions between different gestures could be explained as bifurcations of a nonlinear system having at least two nonlinearly interacting timescales. In addition, applying a cooling technique ${ }^{4,6}$ we could manipulate one of the bifurcations of this system.

These results add up to a series of evidences supporting the "integrative model:" from a theoretical side, detailed topological description of the pressure gestures ${ }^{10,11}$ and their simulation by means of low dimensional dynamical models. ${ }^{12,13}$ From an experimental perspective, the supporting evidence includes simple rhythmic brain stimulation generating respiratory activity ${ }^{5}$ and the breaking of the gestures with a cooling experiment. ${ }^{6}$

The organization of this paper is as follows. In Sec. II, we start describing the importance of the pressure gestures in the generation of vocalizations in birdsong and how we measured them. Then, we describe the naturally occurring bifurcations found in the respiratory gestures of canary song. In Sec. III, we describe the model used and its characteristics, in the perspective of using numerical simulations. We show that the bifurcations that we found may have different origins: due to an amplitude modification or due to a frequency modification of the input forcing activity of the model. We use local temperature manipulation to induce bifurcations. Then, we show a bifurcation map of the model with the paths in parameter space that the birds may use to generate the motor gestures presented in this work. Finally, we present the "Discussion and Conclusions."

\section{PRESSURE GESTURES}

The identification of the physiological gestures involved in avian vocal production required deep studies of the avian vocal organ, the syrinx. ${ }^{14,15}$ The sound is produced there by the vibration of a tissular structure called labia, which are located in the intersection between the bronquia and the trachea. Biomechanical models describing these oscillations using two time dependent parameters (the tension in a ventral syringeal muscle (vS) and the subsyringeal air pressure) allowed to reproduce realistic synthesis of song. ${ }^{16-18}$ These variables can be measured experimentally. In this work, we 
studied the pressure physiological gesture. To measure it, we inserted a cannula in the abdominal air sac of the canaries with the other end connected to a piezoresistive transducer located in a backpack on the bird. ${ }^{14}$ The signal was acquired, after amplifying and filtering it, through a sound card connected to a computer, simultaneously with the song recorded with a microphone. ${ }^{6}$

Pressure gestures used to generate the acoustic syllables can be described and classified from their morphology. Different unit elements can be recognized as the time trace between two consecutive inspirations, where air sac pressure is negative with respect to ambient pressure. From the topological study of low dimensional models, it was recognized that the diverse pressure patterns found in canary song appear as subharmonic solutions of a driven nonlinear system. ${ }^{10-13}$ In addition, identification of two separated timescales interacting nonlinearly in birdsong brain motor pathway gave support to this scheme where classification comes from the different locking regimes between the modeled forcing signal and the output trace. ${ }^{6}$ The mostly found relationships are 1:1, $2: 1$, and 1:1 over a constant value (sustained expiration), which we call $p 1, p 2$, and pulsatile, respectively. In general, $p 1$ syllables are reminiscent to sinusoidal oscillations, $p 2$ have one or two local minima during the expiration, and pulsatiles look like sinusoidal plus a constant. Interestingly, all this diversity can be obtained in the models by changing only two parameters of the forcing signal.
The diversity as a sequence of distinct elements can be understood under a bifurcation paradigm. In the results that follow, we present pressure gestures that may arise from naturally occurring bifurcations and are ubiquitous representative examples of canary singing behavior.

From an acoustic classification birdsong is divided in a succession of syllables which are identified in general with a continuous vocalization. In the case of the canary, they last between 15 and $300 \mathrm{~ms}$ and are repeated at frequencies that go from $3 \mathrm{~Hz}$ to $60 \mathrm{~Hz}$. Each bird has a repertoire of several dozen syllables that combines in bouts that can last for dozens of seconds. We show on top of Figs. 1(a)-1(c) three examples of a song segment sang by a canary with a first part of the pressure pattern with $p 2$ like syllables, followed by simple $p 1$ syllables. In contrast, the sonogram shows a unique syllable type that varies little across repetitions. Within this classification, we find that two acoustically almost identical vocalizations are produced twice in the $p 2$ pressure pattern. From physical modeling of birdsong, we learned that the tension is strongly related with the vocalization frequency, thus suggesting that the principal change across this pattern is in the pressure gesture. ${ }^{2}$ Syllabic length remains constant before and after the transition in the motif and the $p 2$ pattern lasts twice as long as the $p 1$ (Fig. 1(d)). This observation supports a view where the change is due to a smooth modification in some of the physiological instructions controlling respiration, leading to a period halving in the pressure pattern trace. (a)
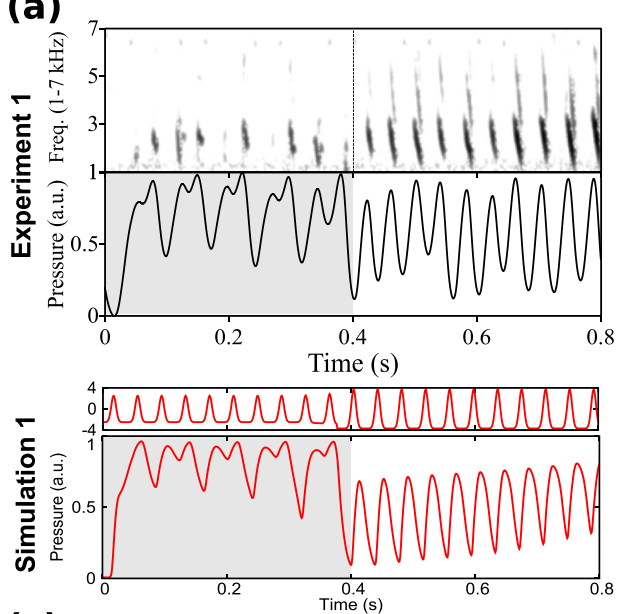

(c)
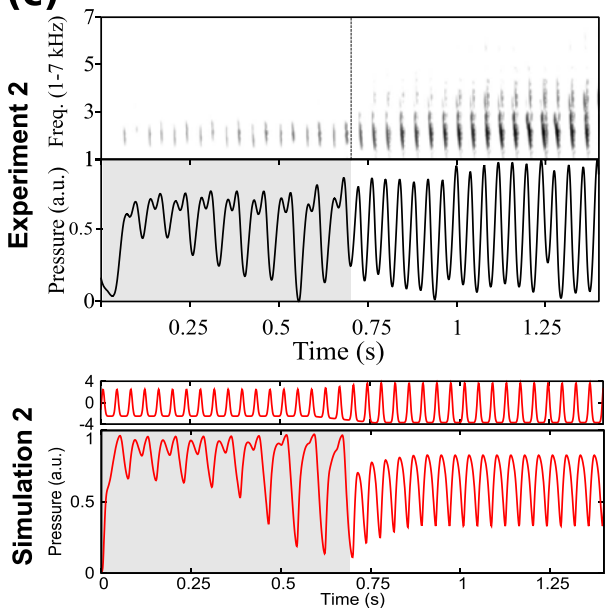

(b)
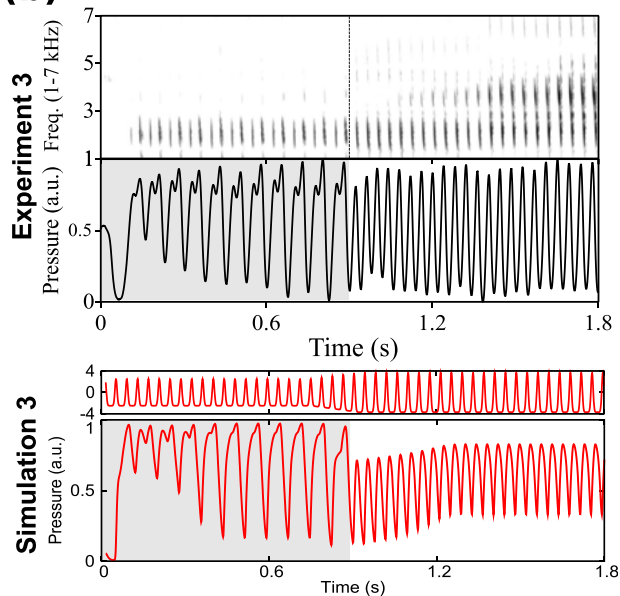

(d)

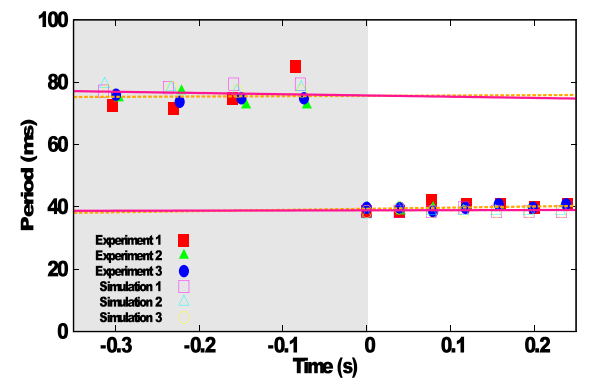

FIG. 1. Naturally occurring bifurcation in the amplitude parameter. (a)-(c) Three examples of syllables sang by a canary that transition naturally between a period 2 syllable (grey shaded) and a period 1 (white). Top experimental panels show the sonogram, top simulation panels the model average activity of the forcing (a.u.) and lower panels show the pressure patterns (a.u.). Acoustical almost identical output occurs twice in each period 2 respiratory pattern. Modeled syllables go through a period 2 bifurcation when the forcing amplitude at $A=2.5$ in a $2: 1$ locking regime is increased linearly $(7 / \mathrm{s})$ until reaching a $1: 1$ regime at $A=3.7$. (d) Period of the syllables against time aligned to the first period 1 syllable. Linear fits before and after transition show a constant syllabic frequency $(f=1 /$ period) before and after transition with 0 slope and a y-intercept halved. Full symbols and line are for experimental data and open symbols and dashed line are for the modeled data. Linear y-intercepts before transition are $(76 \pm 11) \mathrm{ms}$ and $(76 \pm 4) \mathrm{ms}$ for experiment and model, respectively, and $(39 \pm 1) \mathrm{ms}$ after transition for both. 
A distinct syllabic transition was present in another bird, which consists of a pulsatile regime changing into a p2 pattern (Fig. 2(a) top panels). Again, two identical acoustic syllables occur in each $p 2$ pressure gesture, and change between pulsatile syllable and $p 2$ is smooth, being a slight increase in vocalization frequency. Pressure pattern period increases along pulsatile oscillations from $33 \mathrm{~Hz}$ up to $22 \mathrm{~Hz}$ before transition and remains almost constant at $p 2$ regime, approximately doubling its length before and after syllable transition (Fig. 2(b), top). This observation again can be thought as a smooth modification, in this case of the frequency of the physiological instructions controlling respiration, leading to a period doubling in the pressure gesture.

\section{THE MODEL}

A wide range of mechanisms were speculated to give rise to the dynamical origin of the pressure gestures, ranging from the total control of every timescale in the telencepha$\operatorname{lon}^{3,4}$ to other more integratives. ${ }^{5-8}$ In particular, it has been shown that the diversity of gestures can be generated as subharmonics of two nonlinearly interacting timescales. ${ }^{6}$ We show that the song syllables and their transitions can be reproduced with our model and that the changes described can be interpreted as bifurcations in the model. This opens the possibility of interpreting comportamental significant changes with minimal modifications in the physiology.

In order to introduce a plausible mechanism giving rise to one of the interacting timescales in our problem, we start with two neuronal populations. We model the dynamics of their activities by means of an additive neuronal model known as Wilson Cowan ${ }^{19}$ that receives its input as arguments of a saturating function $S(x)=1 /\left(1+e^{x}\right)$ :

$$
\begin{aligned}
\tau \frac{d x}{d t} & =-x+S\left(\rho_{x}+10 x-10 y+F(t / \tau)\right) \\
\tau \frac{d y}{d t} & =-y+S\left(\rho_{y}+10 x+2 y\right),
\end{aligned}
$$

where $x$ is the excitatory activity, $y$ is the inhibitory activity, $\rho$ are basal activity inputs, $\tau$ is a scaling parameter and $F(t / \tau)$ is the forcing activity received from an upstream nucleus. This forcing represents the second timescale in our description. Numbers in the equations are the same as in Ref. 19 , and $\rho_{x}=-2.8$ and $\rho_{y}=-10.7$ is a condition that places the system near a Takens Bogdanov bifurcation, where we can find oscillatory and an on-off type of behavior depending on the values of $F$. This same bifurcation, unfolded in its normal form and forced with an oscillatory $F$, was capable to reproduce all the different types of respiratory gestures found in canary song. ${ }^{12}$ The $x$ activity resulting from the connectivity and the forcing is supposed to project to the motor neurons that innervate the muscles involved in generating the respiratory patterns, so we interpret $x$ to be proportional to the output of the system. The parameter $\tau=0.0088$ was selected to match our simulation frequencies with the ones measured in the respiratory activity in singing canaries.

We provide a plausible interpretation of the parameters and variables of our dynamical model in terms of our animal model. In order to do so, we will briefly describe the neuroanatomy involved in the generation of motor gestures in the songbird brain. Neurons group in different sets called nuclei which are connected by the axonal projections of their neurons, and conform what is called a motor pathway. The birdsong motor pathway has a nucleus called HVC (proper name) which projects to the RA (nucleus Robustus of the archistriatum), which in turn projects to two brainstem structures: nXII (tracheosyringeal part of the hypoglossal nucleus) (a)
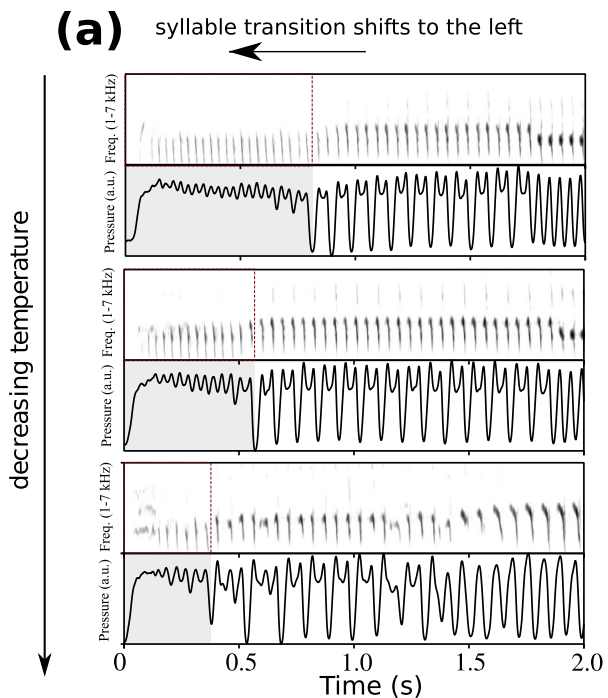

(c)

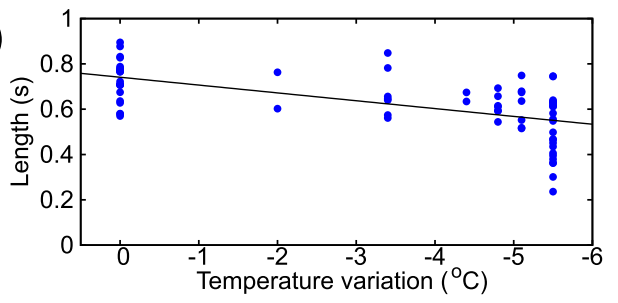

(b)
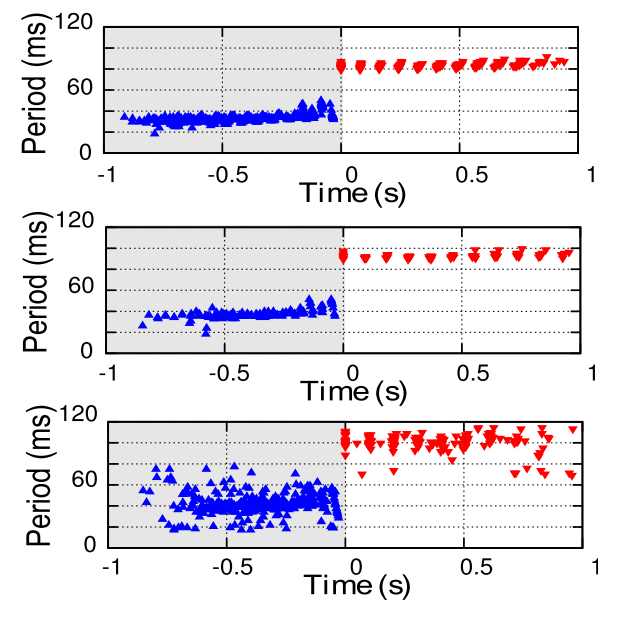$$
1
$$

(n)

FIG. 2. Naturally occurring bifurcation in the frequency parameter is manipulated with temperature. (a) Three examples of experimental song renditions of pulsatile syllables (grey shaded) followed by period 2 syllables (white) at different HVC temperature variations: normal, $-3.4^{\circ} \mathrm{C}$ and $-5.5^{\circ} \mathrm{C}$. As temperature is decreased, the time of regime change is shifted to the left. (b) Syllabic period against time aligned to the first $p 2$ syllable. Period rises with temperature decrease, showing the effect of syllabic frequency reduction of the cooling. There is a naturally occurring period increase before transition. (c) Total duration of pulsatile syllables is decreased with temperature variation in HVC, showing a second effect of cooling: the earlier reach of syllable transition. Tendency line has a slope of $(35 \pm 6) \mathrm{ms} /{ }^{\circ} \mathrm{C}$. Total songs analyzed are 71 across all temperatures. 
which innervates the muscles of the syrinx and RAm (nucleus retroambigualis), which together with PAm (nucleus parambigualis) are in charge of the control of respiration. ${ }^{1}$ Part of this respiratory network (PAm), as well as nucleus DM (dorsal medial nucleus of the midbrain), project back to HVC via the nucleus Uva (nucleus uvaeformis). The dynamics in this recurrent network is affected when HVC is cooled. ${ }^{4,6}$ We model $F$ as an activity of this recurrent network, which acts as a forcing on the coupled system of equations representing two neuronal populations, located downstream in the song system, probably as part of the brainstem nuclei involved in respiration. This model was shown to be a simple architecture necessary for reproducing much of the diversity and complexity in birdsong. ${ }^{6,10-12}$

Average instructions $F$ were modeled with a rhythmic period $T$ in $t \in[-T / 2, T / 2]$ for songs consisting of repeated syllables

$$
F(t)=A \cos (2 \pi f t) N(\nu, \sigma, t),
$$

where $A$ is the amplitude, $f=1 / T$ is the frequency, and $N(\nu, \sigma, t)$ is the normal distribution, centered at $\nu=0$, with a width $\sigma$ of $11.1 \%$ of the period $T$. This gives a width of the neuronal activity of $2 \sigma=8.6 \mathrm{~ms}$ at a frequency of $26 \mathrm{~Hz}$. Supporting our representation, it has been reported in swamp sparrows (Melospiza georgiana) that HVC projection neurons repeat their spiking pattern when a syllable is being repeated. ${ }^{20}$ Although it was reported for neurons projecting to other nucleus (Area X), it is likely that RA-projecting neurons in $\mathrm{HVC}$ also have a long timescale associated with the syllable repetition rate, reflecting stereotyped HVC activation for repeated syllables.

This model displays subharmonicity, with the output periodic pressure patterns locked in a specific relation to the input forcing activity, and results from two nonlinearly interacting timescales, one involving the dynamics of the network including HVC $(F)$, and the other in the nonlinear oscillator ( $x$ and $y$ ).

We simulated the pressure patterns described for the first bird in Sec. II with a fixed frequency of $25.8 \mathrm{~Hz}$ in parameter space that was obtained from the experimental data. We used a amplitude where we found the most similarity between the experiment and simulation in a 2:1 locking regime, $A=2.5$ in a.u (Figs. 1(a)-1(c), bottom). Then we varied only the amplitude with a linear increase until reaching $A=3.7$ where the output is in a 1:1 locking relation with the forcing. We adjusted the "on" and "off" times of the ramp depending on the amount of syllables found before the transition in each song. As a result, we found remarkable morphological similarity between experiment and simulation. We postulate that the origin of this pressure pattern transition is due to a naturally occurring bifurcation in the amplitude of the forcing parameter in the model. Linear fits of the period of the experimental and simulated syllables across time show its values halved and precisely matched between experiment and simulation (Fig. 1(d)). A 0 slope in the fits shows that syllabic frequency does not vary in the transition and although the change in the amplitude of the forcing cannot be directly measured, it could be interpreted as the amount of RA-projecting neurons active in HVC, or as the degree of synchrony between them.
The other analyzed bird, the one with the pulsatile $-p 2$ transition was subjected to a cooling protocol. ${ }^{6}$ We used a cooling device built from a commercial Peltier module, which could decrease locally and in a controlled manner the temperature of a brain region in canaries. A specifically designed gold pad implant was located directly against the brain of the animal bilaterally over the HVC, and seven different local HVC temperatures were obtained while the bird sang its previously described song segment. In Fig. 2(a), second and third joint panels show the song at $\Delta T=-3.4^{\circ} \mathrm{C}$ and $-5.5{ }^{\circ} \mathrm{C}$. The duration of the pulsatile song segment is decreased and for the lowest temperature, a 3:1 regime appears before transitioning to the $p 2$. Pressure gesture period increases for both types of patterns with decreasing temperature (Fig. 2(b), second and third rows). As previously described, ${ }^{4,6}$ cooling has a slowering effect over all timescales of song, which implies that all syllabic frequencies decrease. In addition, we found in our data a shortening of the whole duration of the pulsatile syllables. Since there are natural fluctuations in the number of syllables repeated each time that the canary sings (as we see in Figs. 1(a)-1(c)), we collected many data points to support this observation. We can observe this fluctuation in the vertical spread of points in Fig. 2(c), either in normal conditions $\left(0^{\circ} \mathrm{C}\right.$ Temperature variation) or with a temperature decrease. Time until reaching the transition possibly varies due to statistical physiological fluctuations in the instructions of each bout repetition, but on top of this fluctuation, we can see in Fig. 2(c) a clear tendency across all recorded repetitions for the total pulsatile syllables length to shorten, although the individual syllables last longer (Fig. 2(b)). This can be explained postulating that this is a naturally occurring transition in frequency that was manipulated with the cooling, inducing the reach of a syllabic frequency transition threshold earlier in the bout. Although we cannot rule out some amplitude change in the forcing, a frequency variation is sufficient to explain the effect. This suggests that the time of transition of this naturally occurring bifurcation is not controlled only by a timescale present in HVC, but is rather appearing from its interaction with the rest of the motor pathway.

The results presented before strongly support our suggested picture, where diversity in complex patterns arises from simple instructions. Smooth changes in amplitude and frequency of this forcing instructions resulted in bifurcations in the output pressure patterns. In order to have a broad visualization of these results, we constructed a bifurcation map that we show in Fig. 3(a), for the forcing of Eq. (2). Different locking regimes are denoted with different colors and full arrows represent the path used in the simulation of the patterns shown in Figs. 1 and 2. Bifurcations occur naturally when there is a region change across the path. Period halving occurs in the amplitude parameter when following the vertical arrow that comes from a 2:1 locking regime (green) and ends in a 1:1 (red), which is the path used in Fig. 1. Period doubling occurs in the frequency parameter when following the horizontal arrow from a 1:1 pulsatile locking regime (orange) ending in a 2:1 locking (green), which is a possible path used to generate the patterns of Fig. 2 (Fig. 3(b) shows the simulation for the full arrow). Manipulation 
(a)

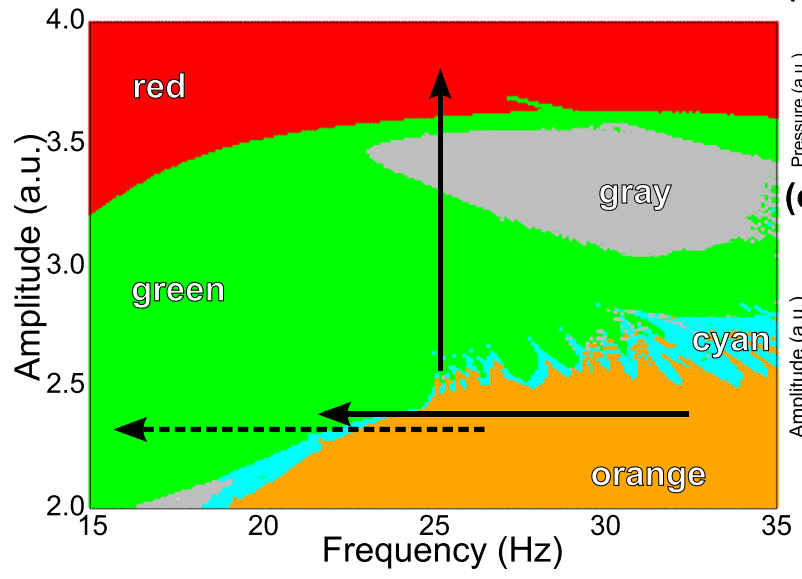

(b)

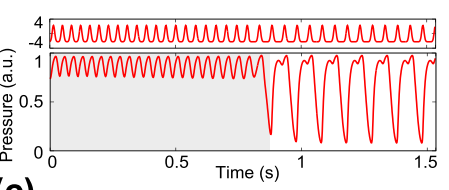

(c)

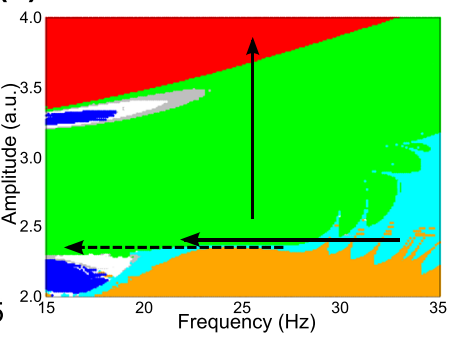

FIG. 3. Locking regimes and bifurcations in canary respiratory gestures. (a) Diagram shows colored regions representing the obtained locking regimes between the forcing frequency and the output pressure pattern. Full vertical and horizontal arrows correspond to the postulated path in parameter space used for the generation of syllables of the canaries of Figs. 1 and 2, respectively. Cooling induces an increase in pattern length, giving slower frequencies and shifting to the left the original values. Dashed arrow shows this effect on the horizontal path: Pulsatile syllable length is shorter, since green region is reached earlier. Bifurcations occur when following an arrow one changes to a different locking region. Red is 1:1 locking, green 2:1, orange 1:1 above constant expiration, cyan 2:1 above constant expiration, grey 4:1. (b) Modeled pressure pattern along the horizontal full arrow path in (a). Starting at $33 \mathrm{~Hz}$ and 2.25 in amplitude, a linear decrease of $7 \mathrm{~Hz} / \mathrm{s}$ is applied in the forcing activity (shown in the upper panel in a.u.), finishing at $22 \mathrm{~Hz}$, same as experimental gesture of Fig. 2. (c) Modified diagram using forcing deltas of constant duration (see Eq. (3)). Here, we find also locking regimes 3:1 in blue and 6:1 in white. Rearrangement of regions retains main bifurcations of the map.

of the frequencies of the horizontal path is represented by the dashed arrow. Cooling HVC, which is supposed to be related to the forcing timescale of the system, reduces its frequency, shifting the parameter accounting for the frequency to the left. This causes that the portion of the dashed arrow (lowest temperature path) in the pulsatile region (orange) is less than for the full horizontal arrow (normal temperature path). This represents a shortening in the time that the parameters stay in this region, and therefore the second type of locking regime is reached earlier in a path with cooling. This explains in a visual way, how our model can account for the counterintuitive shortening in total syllables length for the pulsatile syllable, just before the transition to the $p 2$ syllable.

A second forcing that we explored is one with a fixed width of $2 \sigma=8.6 \mathrm{~ms}$ for every frequency $f$. A single pulse of activity being

$$
F(t)=2 A N(\nu, \sigma, t)-A
$$

again centered at $\nu=0$ and repeated every $T$. The multiplication by $2 A$ and the subsequent subtraction of $A$ modifies only the width of the pulse and not the forcing amplitude qualitative variation that gave the sinusoidal multiplication in Eq. (2). This second type of forcing allows to avoid the non physiological narrowing of the pulses at higher frequencies and retains the most important feature of the forcing, its lowest frequency component $f$. It also represents a step towards modeling song related brain activity in terms of sparse, brief bursts ( $\sim 10 \mathrm{~ms}$ ) present across the song pathway. ${ }^{18}$ In Fig. 3(c), we show how the map is modified with a forcing like Eq. (3).

These maps help visualizing the locking regimes and also the bifurcations present in the model. They present an easy view of how nonlinear interaction between different timescales in a system with simple instructions gives rise to diversity.

\section{DISCUSSION AND CONCLUSIONS}

We measured pressure gestures in canaries, and the acoustic song that is generated with them, and found that transitions between different pressure patterns can be explained with the help of a bifurcation paradigm. This means that the diversity in the output behavior in this motor system can be explained in terms of soft changes in the instructions controlling the physiological behavior.

We used a low dimensional model that allows to reproduce the shape, the times, and the change of the measured patterns having only two parameters. The model helped in the identification of two different mechanisms that give rise to a period doubling bifurcation, the modification of the amplitude, and of the frequency of the forcing instructions. These are interpreted as the parameters that describe the output activity of the network whose dynamics is affected as nucleus HVC is cooled. We did concentrate on the syllable repetition timescale, so we modeled the input into the driven network as a simple periodic pattern (i.e., a sinusoidal narrowed by a Gaussian, or a periodically repeated Gaussian). This simplification corresponds to the lowest frequency present in the Fourier expansion of the signal representing the forcing activity expected when syllables are being repeated. A cooling technique was used to manipulate one of the frequencies needed to generate the measured patterns. With this tool, we obtained a modification in the transition between pressure gestures in a way that is compatible with the observation of naturally occurring bifurcations and the model presented to explain them.

The results reported for zebra finches in the "single timescale" paradigm, ${ }^{4}$ represent the expected stretching changes of an initial cooling, but the syllabic transitions in the songs studied may not be due to bifurcations, or some new bifurcations may be reached with increased cooling. ${ }^{6}$ 
Therefore, the stretching of zebra finch song can be interpreted as a nonlinear locking effect. This is in clear contrast with a widely accepted view of HVC present at the top of a hierarchical structure, with the rest of the pathway merely executing commands given at a $10 \mathrm{~ms}$ timescale. The observation that activity in nuclei in the brainstem can be tracked to the telencephalon already suggested that the whole song pathway (and not just HVC) is involved in determining the temporal features of song. ${ }^{8}$ Here, we show that regardless of the mechanism involved in generating the timescales present in HVC, they can be controlled independently of (at least) a second one. The expiratory gestures emerge as these two timescales interact nonlinearly.

Finally, our results build confidence on a view that considers the motor activity generated by the song system as the result of at least two nonlinearly interacting timescales. The paradigmatic bifurcations encountered represent a characteristic signature of this nonlinear interaction. This could have broad implications in the study complex biological behavior, and, in particular, brain activity, noting that diversity may arise from slight changes of very simple instructions.

\section{ACKNOWLEDGMENTS}

This work was supported by Conicet and the University of Buenos Aires.

${ }^{1}$ H. Zeigler and P. Marler, Neuroscience of Birdsong (Cambridge University Press, 2008).

${ }^{2}$ G. B. Mindlin and R. Laje, The Physics of Birdsong (Springer, New York, 2005).

${ }^{3}$ M. S. Fee, A. A. Kozhevnikov, and R. H. Hahnloser, "Neural mechanisms of vocal sequence generation in the songbird," Ann. N.Y. Acad. Sci. 1016, 153-170 (2004).

${ }^{4}$ M. A. Long and M. S. Fee, "Using temperature to analyse temporal dynamics in the songbird motor pathway," Nature 456, 189-194 (2008).
${ }^{5}$ J. Méndez, G. Mindlin, and F. Goller, "Interaction between telencephalic signals and respiratory dynamics in songbirds," J. Neurophysiol. 107, 2971-2983 (2012).

${ }^{6}$ M. A. Goldin, L. M. Alonso, J. A. Alliende, F. Goller, and G. B. Mindlin, "Temperature induced syllable breaking unveils nonlinearly interacting timescales in birdsong motor pathway," PLoS ONE 8(6), e67814 (2013).

${ }^{7}$ M. Schmidt, J. McLean, and F. Goller, "Breathing and vocal control: The respiratory system as both a driver and target of telencephalic vocal motor circuits in songbirds," Exp Physiol. 97, 455-461 (2012).

${ }^{8}$ R. C. Ashmore, J. A. Renk, and M. F. Schmidt, "Bottom-up activation of the vocal motor forebrain by the respiratory brainstem," J. Neurosci. 28(10), 2613-2623 (2008).

${ }^{9}$ R. H. R. Hahnloser, A. A. Kozhevnikov, and M. S. Fee, "An ultra-sparse code underlies the generation of neural sequences in a songbird," Nature 419, 65-70 (2002).

${ }^{10}$ M. A. Trevisan, G. B. Mindlin, and F. Goller, "Nonlinear model predicts diverse respiratory patterns of birdsong," Phys. Rev. Lett. 96, 058103 (2006).

${ }^{11}$ J. A. Alliende, J. M. Méndez, F. Goller, and G. B. Mindlin, "Hormonal acceleration of song development illuminates motor control mechanism in canaries," J. Dev. Neurobiol. 70, 943-960 (2010).

${ }^{12}$ L. M. Alonso, J. A. Alliende, F. Goller, and G. B. Mindlin, "Low-dimensional dynamical model for the diversity of pressure patterns used in canary song," Phys. Rev. E 79, 041929 (2009).

${ }^{13}$ L. M. Alonso, J. A. Alliende, and G. B. Mindlin, "Dynamical origin of complex motor patterns," Eur. Phys. J. D 60, 361-367 (2010).

${ }^{14} \mathrm{~F}$. Goller and R. A. Suthers, "Role of syringeal muscles in controlling the phonology of bird song," J. Neurophysiol. 76, 287-300 (1996).

${ }^{15} \mathrm{~F}$. Goller and R. A. Suthers, "Role of syringeal muscles in gating airflow and sound production in singing brown thrashers," J. Neurophysiol. 75, 867-876 (1996)

${ }^{16}$ G. B. Mindlin, T. J. Gardner, F. Goller, and R. Suthers, "Experimental support for a model of birdsong production," Phys. Rev. E 68, 041908 (2003).

${ }^{17}$ Y. S. Perl, E. M. Arneodo, A. Amador, F. Goller, and G. B. Mindlin, "Reconstruction of physiological instructions from zebra finch song," Phys. Rev. E 84, 051909 (2011).

${ }^{18}$ A. Amador, Y. S. Perl, G. B. Mindlin, and D. Margoliash, "Elemental gestures dynamics are encoded by song premotor cortical neurons," Nature 495, 59-64 (2013).

${ }^{19}$ F. Hoppensteadt and E. M. Izhikevich, Weakly Connected Neural Networks (Springer-Verlag, New York, 1997).

${ }^{20}$ J. Prather, S. Peters, S. Nowicki, and R. Mooney, "Precise auditory-vocal mirroring in neurons for learned vocal communication," Nature 451, 305-310 (2008). 